\title{
Reaching Adolescent and Young Adult Cancer Patients Through Social Media: Impact of the Photographs of Meaning Program
}

\author{
Megan E. Pailler, PhD, ${ }^{1}$ Lynda K. Beaupin, MD, ${ }^{2}$ Erin Brewer-Spritzer, BS, ${ }^{1}$ Pei C. Grant, PhD, ${ }^{3}$ \\ Rachel M. Depner, MS, ${ }^{3,4, *}$ Kathryn Levy, MSW, ${ }^{3}$ and Kelly E. Tenzek, PhD ${ }^{4, \dagger}$
}

\begin{abstract}
Purpose: This study assessed the feasibility and preliminary efficacy of the Photographs of Meaning Program for Adolescent and Young Adult cancer patients and survivors (POM-AYA).

Methods: POM-AYA is a structured 10-week meaning-based intervention in which participants post photographs and accompanying narratives through a social media platform. Measures of depression, overall quality of life (QoL), and spiritual well-being were assessed on consent (T1), after completing the 10-week intervention (T2) and 2 months' postintervention (T3). Participants also completed a satisfaction questionnaire and followup semi-structured interviews.

Results: Thirty AYA cancer patients and survivors (ages 17-36) were enrolled in the study. At T2, depressive symptoms were significantly lower and QoL was significantly higher compared with T1. These gains were maintained at T3. There were no significant differences in reported spiritual well-being across the study period. Overall, participants reported high rates of study satisfaction in both the survey and qualitative feedback.

Conclusion: POM-AYA appears to be a potentially beneficial, widely accessible intervention in reducing depressive symptoms and increasing QoL in AYA cancer patients and survivors.
\end{abstract}

Keywords: meaning centered psychotherapy, photovoice, social media, depression, quality of life

\section{Background}

A DOLESCENTS AND YOUNG adults with cancer (AYAs) face unique coping challenges due to disruption of key developmental tasks. ${ }^{1}$ AYAs are at risk for a variety of negative psychological outcomes during and after treatment, including depression, anxiety, posttraumatic stress, and fears of recurrence. ${ }^{2-5}$ Despite consistently reported unmet psychosocial needs during and after treatment, ${ }^{1,6}$ AYAs underutilize formal psychological support services. ${ }^{6}$ Research into effective psychosocial interventions for AYAs has been significantly limited by small sample sizes and difficulty enrolling AYAs into traditional studies. ${ }^{7}$

Social media (SM) provides a promising platform for reaching and engaging AYAs as they have a very high rate of usage, with up to $90 \%$ of individuals age 18-29 using SM sites. $^{8}$ A variety of SM platforms exist to provide networking and support opportunities to patients; however, the efficacy of these platforms on improving distress has not been systematically evaluated. There is mixed evidence regarding the provision of internet-based psychological and health promotion interventions to AYAs, with some studies demonstrating higher retention rates compared with traditional methods, and others demonstrating difficulty engaging and retaining AYA participants. ${ }^{9,10}$ Given AYAs' expressed desire for improved social connectedness with other AYAs, ${ }^{11}$ as well as their comfort and familiarity with SM as a communication tool, supportive interventions utilizing SM may be more appealing and less stigmatizing than traditional psychotherapeutic interventions.

The Photographs of Meaning Program (POM) is a novel intervention approach that uses an SM application and platform to deliver a 10-week meaning-based psychosocial intervention to AYAs (POM-AYA). The intervention was designed to incorporate empirically based content into a delivery modality that could more effectively reach and engage AYAs and overcome barriers associated with traditional psychotherapeutic approaches. The format of POM was derived from photovoice, a community-based participatory action research strategy in which participants document and

${ }^{1}$ Department of Psychology, Roswell Park Comprehensive Cancer Center, Buffalo, New York, USA.

${ }^{2}$ Pediatrics, Johns Hopkins All Children's Hospital, Saint Petersburg, Florida, USA.

${ }^{3}$ Palliative Care Institute, The Center for Hospice and Palliative Care, Cheektowaga, New York, USA.

${ }^{4}$ University at Buffalo, The State University of New York, Buffalo, New York, USA.

Present address: Departments of $*$ Counseling and ${ }^{\dagger}$ Communication, School and Educational Psychology, Buffalo, New York, USA. 
share their realities through photographs and discussions around particular themes. ${ }^{12,13}$ Photovoice has been suggested as a mechanism for facilitating dialogue and shared experiences among AYAs, as it allows them a structured mechanism for connecting around areas of shared meaning. ${ }^{14}$ The process of documenting and sharing experiences through posting and interacting around photographs may uniquely resonate with this population given their utilization of SM.

The content of POM was derived from meaning-centered psychotherapy (MCP), a manualized, structured psychotherapeutic intervention designed to help cancer patients sustain and enhance a sense of meaning, purpose, and peace. It incorporates existential concepts such as freedom, responsibility, choice, creativity, identity, and engagement. ${ }^{15}$ $\mathrm{MCP}$ in individual and group contexts has been shown to be an effective treatment in adult cancer populations in reducing psychological and spiritual distress. ${ }^{16}$ It was chosen as the structure of the current intervention given its focus on identity, social connectedness, and hope, which are salient developmental themes associated with this population and the ability to translate its themes into a photograph/narrative structure. POM has been demonstrated in a recent pilot study to be well received by AYA patients and survivors, who rated the content and structure of the program favorably. ${ }^{17} \mathrm{POM}$ has also been piloted among caregivers of pediatric palliative patients, with similar favorable reception. ${ }^{18}$

This study sought to further explore the feasibility and efficacy of the 10-week POM-AYA program. Feasibility was defined in terms of retention rates in the study as well as feedback from participants. We hypothesized that completion of the 10 -week intervention would result in decreased depressive symptoms, improved spiritual well-being, and improved social and psychological aspects of quality of life (QoL), both at completion of the program and at the 2-month follow-up.

\section{Methods}

\section{Design}

This study was an exploratory mixed-method cohort design that examined the feasibility and efficacy of POM-AYA on psychological well-being in an AYA cancer population. The study was approved by the Comprehensive Cancer Center's Institutional Review Board (I-44717).

\section{Participants}

Participants were identified through our Cancer Center's Clinical Data Network (CDN), a center-specific disease registry of all patients diagnosed. The study was run in two cohorts to reduce time between enrollment and study launch. Preliminary data from cohort one has been previously reported. ${ }^{17}$ Recruitment was through a direct mailing and follow-up phone calls regarding the nature and purpose of the study. The CDN identified 450 potential participants. Participants with valid phone numbers were then contacted by phone to assess eligibility and interest. Eligibility criteria included AYA cancer patients and survivors who were between 15 and 34 years of age at the time of diagnosis, diagnosed within 3 years, English speaking and who had received treatment for their disease (e.g., chemotherapy, radiation, surgery). Due to technological requirements for the study, participants were excluded if they did not possess a smart- phone, tablet, or computer with camera and Wi-Fi capability, or a valid email address. Patients with significant cognitive impairments, dementia, or untreated severe and persistent mental illness were also excluded from participation. Prospective participants attended a one-time, in-person or remote consenting session, where study risks and benefits were explained. Participant recruitment and consent sessions occurred over an $\sim 3$-month timeframe for each cohort.

\section{Procedure}

After informed consent, participants were given access to the mobile application, PixStori ${ }^{\mathrm{TM}}$, and provided with brief training, including how to set up a user profile and how to record and upload Pixstories. Participants were administered a series of self-report questionnaires before the start of the intervention (T1), after the 10 weeks (T2), and 2 months after completing the intervention (T3). On completion of the 10-week intervention, participants provided feedback via questionnaire and phone interview.

\section{Measures}

Depressive symptoms. Depressive symptoms were assessed by using the Beck Depression Inventory-II (BDI-II), a 21item, self-report measure of depressive symptoms that has been widely used and validated with adolescent and adult populations. ${ }^{19}$ Items on the BDI-II are rated on a 4-point scale (ranging $0-3$ ) based on severity, with a maximum total score of 63 . This study utilized electronic administration of the BDI-II through the Pearson Q-Global platform. Internal consistency estimates are in the $\alpha=0.9$ range, and test-retest reliability ranges from 0.73 to 0.96 in a wide range of samples. ${ }^{19}$ The BDI-II has demonstrated good convergent and discriminant validity. ${ }^{19}$

Quality of life. QoL was assessed with the PedsQL-YA, a 23-item self-report measure consisting of a total score (23 items), a physical health composite ( 8 items), and a psychosocial health composite (13 items). ${ }^{20}$ Items on the PedsQL are rated on a 5-point scale ranging from 0 (Never) to 4 (Almost Always). Scores on the PedsQL-YA are reversed and transformed to a $0-100$ scale, where higher scores indicate better health-related QoL. ${ }^{20}$ This QoL measure is one of a very few that has been validated across the AYA age range used in this study. The PedsQL-AYA has good internal consistency (Cronbach's $\alpha$ ranging from 0.81 to 0.98 ), and it demonstrates clinical validity in its ability to differentiate among groups with mild, moderate, and severe symptoms ${ }^{20}$ For this study, the total score was used as the measure of overall QoL.

Spiritual well-being. Spiritual well-being was assessed with the FACIT-Sp, a 12-item self-report measure assessing aspects of spiritual well-being such as peace, meaning, and faith that are not limited to any specific religion or faith. ${ }^{21}$ Items on the FACIT-SP-12 are rated on a 5-point scale ranging from 0 (not at all) to 4 (very much). The FACIT-Sp has good internal consistency (Cronbach's $\alpha$ ranging from 0.81 to 0.88 ), and it correlates moderately with other measures of QoL and spiritual well-being. ${ }^{21}$

Participant satisfaction. Participant satisfaction was assessed via satisfaction surveys and semi-structured interviews completed at $\mathrm{T} 2$. Using a 5-point Likert-type scale, 
participants rated the overall helpfulness of the intervention as well as specific aspects such as content of weekly themes, cohort size, usability of the Pixstori application, and preferences regarding audio narration versus text. They also provided qualitative descriptions of the aspects of the intervention that were most helpful, as well as aspects that they would have changed or added.

Participant baseline demographic and clinical data (i.e., cancer diagnosis, treatment complexity) were collected during the initial eligibility phone screen. All questionnaires were presented to participants remotely through REDCap and Pearson Q-Global applications.

\section{SM application: PixStori}

All participants were given access to the study portal on the PixStori mobile and website application. PixStori was chosen as the platform for this study due to its unique ability to function as a secure remote SM platform by which participants were able to upload photographs and audio record or text narratives based on their interpretations of the weekly themes. Participants posted their "stories" to the cohort portal where they could be viewed by other participants. As with other SM platforms, participants could "like" and comment on their own or others' stories, allowing for communication among participants regarding posted content.

\section{Intervention}

The intervention content was adapted from MCP, an empirically supported manualized psychotherapy for advanced cancer patients developed by Breitbart and colleagues. ${ }^{16}$ Content areas of the manualized treatment were adapted by the research team for an AYA population and converted into weekly themes (Table 1). Each week, participants were sent an email or text message introducing them to the weekly theme. Participants were instructed to take or upload to the PixStori study portal at least two pictures per week with their interpretation of the weekly themes. Participants were instructed to use the audio or text narration feature to describe their chosen pictures and how it related to that week's theme. Participants who did not post by mid-week were sent an additional email and text message prompt. All participants were provided a copy of the study's "community standards" and guidelines of

TABle 1. WeEkly Themes

\begin{tabular}{cl}
\hline Week & \multicolumn{1}{c}{ Theme } \\
\hline 1 & Who am I? \\
2 & Living with cancer: How has cancer changed \\
& who I am? \\
3 & What matters most? \\
4 & Understanding my roots or history \\
5 & What makes me proud? \\
6 & Coping with bad days \\
7 & Living life to the fullest \\
8 & Experiencing the moment (Love, Beauty, Humor) \\
9 & My story of uncertainty and hope \\
10 & Review week: What picture stands out the most? \\
\hline
\end{tabular}

Sample prompt questions:

(1) Tell us why you chose the picture.

(2) Tell us how it tells the story of (theme). appropriateness at the time of their consenting session. Research personnel monitored the study portal daily for any graphic or inappropriate content that violated study standards.

\section{Statistical analysis}

Data were analyzed by using SPSS v. 21. Data from both intervention cohorts were combined in all analyses. Preliminary analyses were conducted to assess for normalcy of distribution and the presence of outliers. Feasibility was assessed by examining rates of participation and retention in the study. Participant demographic information was assessed by utilizing descriptive statistics and frequencies as appropriate. Impact of the intervention on study outcomes was assessed with a series of repeated-measures ANOVAs by using time as the withinsubjects independent variable, comparing outcome scores across study points. Follow-up paired $t$-tests were then conducted for significant main effects to examine significant change from $\mathrm{T} 1$ to $\mathrm{T} 2$ and $\mathrm{T} 2$ to $\mathrm{T} 3$. All the tests were two tailed at significance level 0.05. Descriptive statistics from patient satisfaction surveys, as well as a summary of semi-structured interviews are also reported. Qualitative data from the interviews were transcribed and de-identified. Interview transcripts were then read by two study personnel and coded for themes associated with intervention satisfaction and areas for improvement. Any discrepancies were discussed until consensus was reached.

\section{Results}

\section{Participant demographics}

Recruitment letters were sent to 450 participants, 230 of whom received follow-up recruitment calls. Of these, 30 declined participation, 6 were ineligible, and 30 agreed to participate. The majority of potential participants did not respond to messages or did not have valid numbers or voicemail. Thirty participants were enrolled in the study. Twenty-nine participants completed T1 measures, as one participant did not complete T1 measures and never downloaded the PixStori application. At T2, 14 participants completed all outcome measures and 16 participants completed a portion of measures. At T3, 12 participants completed all outcome measures and 13 participants completed a portion of measures. The mean age of participants was 28.23 years $(\mathrm{SD}=5.29$, range 17-36). Full demographic characteristics of the study sample, including diagnosis information and treatment type, are presented in Table 2. Preliminary data analysis revealed significant negative skew for PedsQL. As such, scores were reflected and then transformed by using square root. Descriptive statistics are presented for the untransformed scores for ease of interpretation. Results are presented separately by outcome measure.

\section{Intervention participation and retention}

Of the 30 enrolled participants, 7 (23\%) did not participate in the intervention. Fifteen participants $(50 \%)$ completed at least 9 out of the 10-week intervention, and 8 participants (27\%) had partial completion of the intervention, with typical patterns involving participation in early weeks and then discontinuing posts. One participant engaged in sporadic posts, completing $50 \%$ of weekly assignments. Thus, three general patterns of participation were seen: those with neartotal participation ("full intervention completers"), those 
Table 2. Demographic Characteristics

\begin{tabular}{|c|c|c|c|c|}
\hline Variables & $\begin{array}{c}\text { Full intervention } \\
\text { completers }(\mathrm{n}=15)\end{array}$ & $\begin{array}{l}\text { Intervention non/partial } \\
\text { completers }(\mathrm{n}=15)\end{array}$ & All $(\mathrm{n}=30)$ & $X^{2} p$-value \\
\hline Sex & & & \multicolumn{2}{|c|}{$p=0.031^{\mathrm{a}}$} \\
\hline Males & $3.3 \%(1)$ & $20 \%(6)$ & \multirow{2}{*}{\multicolumn{2}{|c|}{$\begin{array}{l}23.3 \%(7) \\
76.7 \%(23)\end{array}$}} \\
\hline Females & $46.7 \%(14)$ & $30 \%(9)$ & & \\
\hline Age & & & \multicolumn{2}{|c|}{$p=0.439$} \\
\hline $15-25$ & $13.3 \%(4)$ & $20.0 \%(6)$ & \multirow{2}{*}{\multicolumn{2}{|c|}{$\begin{array}{l}33.3 \%(10) \\
667 \%(20)\end{array}$}} \\
\hline$>25$ & $36.7 \%(11)$ & $30.0 \%(9)$ & & \\
\hline Race & & & \multicolumn{2}{|c|}{$p=0.624$} \\
\hline White/Non-Hispanic & $40.0 \%(12)$ & $43.3 \%(13)$ & \multicolumn{2}{|c|}{$83.3 \%(25)$} \\
\hline Other racial groups & $10.0 \%(3)$ & $6.7 \%(2)$ & \multirow{5}{*}{\multicolumn{2}{|c|}{$\begin{array}{r}16.7 \%(5) \\
6.7 \%(2) \\
3.3 \%(1) \\
0 \%(0) \\
6.7 \%(2)\end{array}$}} \\
\hline Black/African American & & & & \\
\hline American Indian/Alaskan Native & & & & \\
\hline Asian/Pacific Islander & & & & \\
\hline Latino/Hispanic & & & & \\
\hline Diagnosis & & & \multicolumn{2}{|c|}{$p=0.587$} \\
\hline Leukemia/lymphoma & $16.7 \%(5)$ & $23.3 \%(7)$ & \multicolumn{2}{|c|}{$40 \%(12)$} \\
\hline Papillary thyroid carcinoma & $13.3 \%(4)$ & $6.7 \%(2)$ & \multicolumn{2}{|l|}{$20 \%(6)$} \\
\hline Cervical & $10.0 \%(3)$ & $3.3 \%(1)$ & \multicolumn{2}{|l|}{$13.3 \%(4)$} \\
\hline Melanoma & $3.3 \%(1)$ & $6.7 \%(2)$ & \multicolumn{2}{|l|}{$10 \%(3)$} \\
\hline Breast & $6.7 \%(2)$ & $3.3 \%(1)$ & \multicolumn{2}{|l|}{$10 \%(3)$} \\
\hline Brain & $0.0 \%(0)$ & $3.3 \%(1)$ & \multicolumn{2}{|l|}{$3.3 \%(1)$} \\
\hline Sarcoma & $0.0 \%(0)$ & $3.3 \%(1)$ & \multicolumn{2}{|l|}{$3.3 \%(1)$} \\
\hline Treatment type & & & \multicolumn{2}{|c|}{$p=0.181$} \\
\hline Combination & $26.7 \%(8)$ & $10.0 \%(3)$ & \multicolumn{2}{|c|}{$36.7 \%(11)$} \\
\hline Chemotherapy & $10.0 \%(3)$ & $23.3 \%(7)$ & \multicolumn{2}{|l|}{$33.3 \%(10)$} \\
\hline Surgery only & $13.3 \%(4)$ & $13.3 \%(4)$ & \multicolumn{2}{|l|}{$26.7 \%(8)$} \\
\hline Radiation & $0.0 \%(0)$ & $3.3 \%(1)$ & \multicolumn{2}{|c|}{$3.3 \%(1)$} \\
\hline Distance to center & & & \multicolumn{2}{|c|}{$p=0.815$} \\
\hline$<15$ miles & $20.0 \%(6)$ & $23.3 \%(7)$ & \multicolumn{2}{|c|}{$43.3 \%(13)$} \\
\hline$>15$ and $<100$ miles & $23.3 \%(7)$ & $23.3 \%(7)$ & \multicolumn{2}{|l|}{$46.7 \%(14)$} \\
\hline$>100$ miles & $6.7 \%(2)$ & $3.3 \%(1)$ & \multicolumn{2}{|l|}{$10.0 \%(3)$} \\
\hline
\end{tabular}

${ }^{\mathrm{a}} p<0.05$.

who did not participate at all, and those who dropped out after 1-2 weeks of participation ("intervention non/partial completers"). The latter two categories were combined for analytic purposes due to small sample size. Study sample demographic characteristics broken down by pattern of participation are presented in Table 2. Males were significantly more likely than females to not complete or partially complete the intervention $\left[X^{2}(1, n=30)=4.66, p<0.05\right]$. There were no other significant differences between the two patterns of participation in terms of age, race, diagnosis, and treatment type, or participant proximity to cancer center.

\section{Impact of intervention on study outcomes}

Descriptive statistics for study outcome measures are presented in Table 3. A series of repeated-measures ANOVAs was performed to assess the impact of the intervention on study outcome measures, including depressive symptoms, QoL, and spiritual well-being. There was a significant main effect of time on depressive symptoms $[F(2,24)=4.87$, $\left.p=0.02, \eta_{\mathrm{p}}^{2}=0.29\right]$ with follow-up paired t-tests, indicating a significant reduction in BDI scores from $\mathrm{T} 1$ to $\mathrm{T} 2$, $[t(12)=2.48, p=0.03]$ and $\mathrm{T} 1$ to $\mathrm{T} 3[t(12)=2.94, p=0.01]$, and no significant difference from $\mathrm{T} 2$ to $\mathrm{T} 3[t(12)=-0.08$, $p=0.94]$. There was also a significant main effect of time on QoL $\left[F(2.22)=7.56, p=0.003, \eta_{\mathrm{p}}^{2}=0.41\right]$, with follow-up paired t-tests indicating a similar pattern of significant improvement in QoL from T1 to T2 $[t(11)=2.85, p=0.02]$ and $\mathrm{T} 1$ to $\mathrm{T} 3[t(11)=4.25, p<0.01]$ and no significant differences from $\mathrm{T} 2$ to $\mathrm{T} 3[t(11)=-1.09, p=0.30]$. There was not a significant main effect of time on spiritual QoL $[F(2,22)=1.15$, $\left.p=0.34, \eta_{\mathrm{p}}^{2}=0.09\right]$.

Table 3. Means and Standard Deviations of Outcome Variables at Baseline and Follow-Up

\begin{tabular}{llll}
\hline & $T 1$ & \multicolumn{1}{c}{$T 2$} & $T 3$ \\
\hline Depression & $57.23(10.35)$ & $51.85(8.53)$ & $52.00(10.14)$ \\
Quality of life & $74.42(13.71)$ & $81.73(14.88)$ & $80.83(14.11)$ \\
Spiritual well-being & $36.58(6.75)$ & $38.17(5.73)$ & $38.75(4.83)$ \\
\hline
\end{tabular}




\section{Participant satisfaction}

Participant satisfaction data were obtained from 17 participants, 15 of whom were intervention completers. Overall participant feedback was very positive, with $94 \%$ of respondents describing the experience as positive or very positive. Themes identified from qualitative interview data included a sense of connectedness with others who have shared experiences, enjoyment of the weekly themes, and knowledge that others struggle with similar issues. The most consistent negative feedback from both surveys and qualitative data involved the functionality and flexibility of the application itself, with $71 \%$ of respondents reporting challenges with the application. The two noncompleters who provided feedback cited health concerns and not enough time as reasons for not participating. Regarding preferences for typing versus audio-recording the weekly assignments, $41 \%$ of participants indicated a preference for texting responses, and $47 \%$ described being comfortable or very comfortable with audio-recording narratives. Of note, during the semi-structured interviews, several participants indicated that they found the audio recordings more meaningful, but they were more likely to read the text than listen to the audio given the time involved and the necessity of privacy or headphones for the audio narratives.

\section{Discussion}

This study sought to assess the feasibility and preliminary efficacy of a 10-week, meaning-centered SM intervention for AYA cancer patients and survivors. AYA populations have been challenging to engage in psychosocial interventions, despite significant evidence of unmet psychosocial needs in this population. Our feasibility results indicate engagement rates of 40-50\% among consented participants. Among those who did participate, engagement rates were high, with very few partial completers. Intervention feedback among participators was very encouraging, with participants often describing the experience as highly meaningful to them. Unfortunately, those participants who did not complete the intervention were also less likely to provide feedback, and, as such, relatively limited information is available from those who dropped out. Of those who did provide feedback, the most frequent reasons involved time commitment, illness burden, or feeling that they did not belong to the target population (either being too sick or too well). We did not collect information on whether the noncompleters continued to passively observe posts throughout the study and if doing so had any benefits. As the majority of SM users are believed to be "lurkers" who never contribute, ${ }^{22}$ methods of measuring passive participation will be important for future studies utilizing SM platforms.

Our intervention also showed promising preliminary evidence of efficacy. Participants endorsed significantly less depressive symptoms and improved QoL at the completion of the study compared with baseline, and these effects were generally preserved at the 2-month follow-up. Effect sizes were generally large and were consistent with participants' qualitative feedback of improved connectedness and a sense of community. The nonsignificant changes in spiritual QoL may reflect the lack of salience of spirituality in this population, or the small sample and relatively small effect sizes of change in this domain. Given the relative lack of information about the role of spirituality in AYAs, it is also possible that spirituality-based measures may not be as well suited in an AYA population to capture meaning-making.

One of the significant strengths of the POM methodology is its ability to reach a large network of AYAs remotely, thus possibly removing geographic barriers to psychosocial treatment. As more than one-third of AYAs in the United States receive their cancer treatment outside of academic centers, ${ }^{23}$ POM-AYA shows promise in providing empirically based, accessible psychosocial support to all AYAs regardless of type of or proximity to treatment site. In addition, the large effect sizes observed in this study provide promising preliminary data on the potential effectiveness of the intervention. One consideration moving forward is how explicitly to adopt additional SM components and structures into the intervention. Feedback from participants indicated that they enjoyed the SM features (e.g., the ability to "like" and comment on others' posts) and requested additional SM features such as notifications of comments on their posts. Conversely, recent concerns regarding privacy and manipulation associated with SM were reflected in some potential participants' refusal to participate and may be more of an issue with interventions utilizing more popular SM platforms.

\section{Study limitations}

There are several limitations to the study. Our sample size was smaller than intended due to technical challenges requiring redesign of the PixStori application, the timing of which prevented additional cohorts within the study period. The relatively low rate of enrollment among eligible patients reflects the well-documented difficulty of engaging this population in research studies. ${ }^{24-27}$ Suggestions for increasing recruitment have involved reaching out to AYAs through SM and other online resources. Interestingly, although this project involved an online format for intervention delivery, we utilized traditional methods of recruitment, including mailed letters and follow-up phone calls, which were negatively impacted by frequently changing phone numbers and addresses associated with AYAs. Future iterations may benefit from accessing AYAs through existing online support communities and social networks. In addition, the lack of control group limits conclusions about the role of the intervention in causing changes in outcome variables, although the stability of changes from T2 to T3 is encouraging. Finally, eligibility requirements such as access to a smartphone or web browser may have limited study participation. Largerscale, randomized controlled studies are needed to further determine what effects are specifically related to the intervention. Future studies would benefit from a broader recruitment strategy, including partnering with existing online AYA support communities and networks for recruitment.

Overall, results of this study suggest that POM shows promise as a novel and cost-effective psychosocial intervention for AYAs. The utilization of pictures as a forum for meaning and connectedness provides a unique forum for provision of a structured, theoretically derived intervention that can be widely disseminated. Future, larger-scale controlled trials are warranted to determine its efficacy.

\section{Acknowledgments}

The authors are grateful to Michael Fritch and Michael Haller of Talking Pictures, LLC who provided technical 
support and modifications to PixStori for this study. Thanks are due to Eric Kishel for assistance in data collection, data entry, and protocol implementation.

\section{Author Disclosure Statement}

No competing financial interests exist.

\section{Funding Information}

This work was supported by an award from the Roswell Park Alliance Foundation and by NCI grant P30 CA016056.

\section{References}

1. Barnett M, McDonnell G, DeRosa A, et al. Psychosocial outcomes and interventions among cancer survivors diagnosed during adolescence and young adulthood (AYA): a systematic review. J Cancer Surviv. 2016;10:814-31.

2. Kwak M, Zebrack BJ, Meeske KA, et al. Prevalence and predictors of post-traumatic stress symptoms in adolescent and young adult cancer survivors: a 1-year follow-up study. Psychooncology. 2013;22(8):1798-806.

3. Seitz DC, Besier T, Debatin KM, et al. Posttraumatic stress, depression and anxiety among adult long-term survivors of cancer in adolescence. Eur J Cancer. 2010;46(9):1596-606.

4. Smith AW, Bellizzi KM, Keegan TH, et al. Health-related quality of life of adolescent and young adult patients with cancer in the United States: the Adolescent and Young Adult Health Outcomes and Patient Experience study. J Clin Oncol. 2013;31(17):2136-45.

5. Bellizzi KM, Smith A, Schmidt S, et al. Positive and negative psychosocial impact of being diagnosed with cancer as an adolescent or young adult. Cancer. 2012; 118(20):5155-62.

6. Zebrack BJ, Block R, Hayes-Lattin B, et al. Psychosocial service use and unmet need among recently diagnosed adolescent and young adult cancer patients. Cancer. 2013; 119(1):201-14.

7. Richter D, Koehler M, Friedrich M, et al. Psychosocial interventions for adolescents and young adult cancer patients: a systematic review and meta-analysis. Crit Rev Oncol Hematol. 2015;95(3):370-86.

8. Perales MA, Drake EK, Pemmaraju N, Wood WA. Social media and the adolescent and young adult (AYA) patient with cancer. Curr Hematol Malig Rep. 2016;11(6):449-55.

9. Bradford NK, Chan RJ. Health promotion and psychological interventions for adolescent and young adult cancer survivors: a systematic literature review. Cancer Treat Rev. 2017;55:57-70.

10. Devine KA, Viola AS, Coups EJ, Wu YP. Digital health interventions for adolescent and young adult cancer survivors. JCO Clin Cancer Inform. 2018;2:1-15.

11. Zebrack B, Bleyer A, Albritton K, et al. Assessing the health care needs of adolescent and young adult cancer patients and survivors. Cancer. 2006;107(12):2915-23.

12. Wang C, Burris MA. Photovoice: concept, methodology, and use for participatory needs assessment. Health Educ Behav. 1997;24(3):369-87.

13. Wang CC, Redwood-Jones YA. Photovoice ethics: perspectives from Flint Photovoice. Health Educ Behav. 2001; 28(5):560-72.
14. Niepage M, Georgievski G, Shama W, Lucchetta S. Exploring adolescents' cancer journey through photovoice: a narrative synthesis. J Adolesc Young Adult Oncol. 2018;7(1):15-21.

15. Breitbart WSaP, S.R. Individual meaning-centered psychotherapy for patients with advanced cancer. New York, NY: Oxford University Press; 2014.

16. Breitbart W, Rosenfeld B, Pessin H, et al. Meaningcentered group psychotherapy: an effective intervention for improving psychological well-being in patients with advanced cancer. J Clin Oncol. 2015;33(7):749-54.

17. Beaupin LK, Pailler ME, Brewer-Spritzer E, et al. Photographs of meaning: a novel social media intervention for adolescent and young adult cancer patients. Psychooncology. 2019;28(1):198-200.

18. Levy K, Grant PC, Depner RM, et al. The photographs of meaning program for pediatric palliative caregivers: feasibility of a novel meaning-making intervention. Am J Hosp Palliat Care. 2019;36(7):557-63.

19. Beck AT, Steer RA, Brown GK. Manual for the beck depression inventory-II. San Antonio, TX: Psychological Corporation; 1996.

20. Ewing JE, King MT, Smith NF. Validation of modified forms of the PedsQL generic core scales and cancer module scales for adolescents and young adults (AYA) with cancer or a blood disorder. Qual Life Res. 2009;18(2):231-44.

21. Peterman AH, Fitchett G, Brady MJ, et al. Measuring spiritual well-being in people with cancer: the functional assessment of chronic illness therapy-Spiritual Well-being Scale (FACIT-Sp). Ann Behav Med. 2002;24(1):49-58.

22. van Mierlo T. The $1 \%$ rule in four digital health social networks: an observational study. J Med Internet Res. 2014; 16(2):e33.

23. Parsons HM, Harlan LC, Schmidt S, et al. Who treats adolescents and young adults with cancer? A report from the AYA HOPE study. J Adolesc Young Adult Oncol. 2015; 4(3):141-50.

24. Cantrell MA, Conte T, Hudson M, et al. Recruitment and retention of older adolescent and young adult female survivors of childhood cancer in longitudinal research. Oncol Nurs Forum. 2012;39(5):483-90.

25. Tonorezos ES, Oeffinger KC. Research challenges in adolescent and young adult cancer survivor research. Cancer. 2011;117(10 Suppl):2295-300.

26. Tercyak KP, Donze JR, Prahlad S, et al. Identifying, recruiting, and enrolling adolescent survivors of childhood cancer into a randomized controlled trial of health promotion: preliminary experiences in the Survivor Health and Resilience Education (SHARE) Program. J Pediatr Psychol. 2006;31(3):252-61.

27. Rabin C, Horowitz S, Marcus B. Recruiting young adult cancer survivors for behavioral research. J Clin Psychol Med Settings. 2013;20(1):33-6.

Address correspondence to: Megan E. Pailler, PhD

Department of Psychology

Roswell Park Comprehensive Cancer Center Elm \& Carlton Street Buffalo, NY 14263

USA

Email: megan.pailler@ roswellpark.org 